\title{
A case of early gastric cancer with a single giant lymph node metastasis
}

\author{
Masato Yoshikawa ${ }^{1 *}$, Misaki Tamario ${ }^{1}$, Masayoshi Obatake ${ }^{1}$, Koichi Sato ${ }^{1}$, Shigehiko Yagi ${ }^{1}$, Hiromi Otani ${ }^{1}$ and \\ Katsumi Kito ${ }^{2}$
}

\begin{abstract}
Background: Early gastric cancer (EGC) is often associated with lymphatic metastasis, but it is extremely rare to be found as a single giant lymph node. Cancer often becomes more malignant in metastatic lesions than in primary lesions, and retrodifferentiation to the fetal gastrointestinal tract during the metastatic process has been reported in gastric cancer. We report an extremely rare case of EGC with a 13-cm giant lymph node metastasis in which an adenocarcinoma with enteroblastic differentiation and yolk sac tumor-like components was observed.

Case presentation: The case was a 70-year-old man who visited his local doctor with right hypochondrial pain, which was identified by computed tomography (CT) as a giant mass. Upper endoscopy revealed a 30-mm-sized 0-Ilc lesion in the greater curvature of the angular incisure and a 15-mm-sized 0-lla lesion in the anterior wall of the lower body of the gastric body. Endoscopic biopsy revealed tubular adenocarcinoma in both lesions. The gastric lesion and the giant tumor were clinically regarded as independent lesions (gastrointestinal stromal tumor, [GIST], and EGCs), and distal gastrectomy and D1 + dissection were performed to comprehensively treat all lesions. Pathological examination revealed that the giant tumor was tubular adenocarcinoma with an intestinal phenotype and was considered a lymph node metastasis of EGCs. To exclude the possibility of metastasis of adenocarcinoma other than EGCs, postoperative positron emission tomography-computed tomography (PET-CT) and colonoscopy were performed; however, no primary site other than the stomach was found. Metastatic lymph nodes have an increased degree of atypia compared with the primary tumor, and yolk sac tumor-like carcinoma morphology was observed along with a-fetoprotein (AFP) and Spalt-like 4 (SALL4) expression in this case. It was considered that retrodifferentiation to a fetal phenotype occurred during the metastatic process. Liver metastasis occurred 6 months after surgery, and chemotherapy is currently being introduced.

Conclusions: We experienced a case of EGC with a single giant lymph node metastasis. Retrodifferentiation to the fetal gastrointestinal tract during metastasis was speculated to be involved in the formation of giant lymph node metastasis and liver metastasis in this case.
\end{abstract}

Keywords: Early gastric cancer, Giant lymph node metastasis, Retrodifferentiation, Yolk sac tumor-like carcinoma

\section{Background}

The frequency of lymph node metastasis in early gastric cancer (EGC) is approximately $16.9 \%$ in submucosal layer (SM) cancer [1]. However, EGC with a single giant

\footnotetext{
*Correspondence: masato08122003@gmail.com

1 Institute of Ehime Prefectural Central Hospital, Department of Digestive

Surgery, Matsuyama, Ehime 790-0024, Japan

Full list of author information is available at the end of the article
}

lymph node metastasis of more than $5 \mathrm{~cm}$ is extremely rare. It is difficult to diagnose lymph node metastasis preoperatively, and in past reports, there have been no cases that giant tumor was diagnosed as lymph node metastasis from EGC, and many cases were diagnosed as gastric submucosal tumors or pancreatic tumors [2]. The phenomenon that cancer becomes more malignant in metastatic lesions than in primary lesions is often experienced, and retrodifferentiation to the fetal gastrointestinal tract 
during the metastatic process also occurs in gastric cancer $[3,4]$. In this case, we experienced a case with a $13 \mathrm{~cm}$ single giant lymph node metastasis of EGC. We report here an extremely rare case of EGCs with a giant lymph node metastasis in which adenocarcinoma with enteroblastic differentiation and yolk sac tumor-like component was observed.

\section{Case presentation}

A 70-year-old man visited his doctor because of right hypochondrial pain, and a giant tumor in the abdomen was identified on computed tomography (CT). In addition, two EGCs were revealed by upper endoscopy, and he was referred to our department. His medical history was type 2 diabetes mellitus and postoperative state of appendectomy, and he had no family history. His height was $158 \mathrm{~cm}$, his weight was $59.7 \mathrm{~kg}$, and his BMI was 23.9, and he had a fist-sized palpable elastic hard mass on the right abdomen. Laboratory tests showed low levels of albumin $(3.4 \mathrm{~g} / \mathrm{dL})$ and hemoglobin $(10.6 \mathrm{~g} / \mathrm{dL})$, and high levels of carcinoembryonic antigen (CEA) $(18.9 \mathrm{ng} / \mathrm{mL})$. Upper endoscopy revealed a 30-mm-sized 0-IIc lesion in the anterior wall of the angular incisure and a $15-\mathrm{mm}$ sized 0-IIa lesion in the lower anterior wall of the gastric corpus (both tub2). A submucosal tumor-like ridge in the lower body of the stomach was suspected to be an exclusion by the aforementioned giant tumor (Fig. 1).

Abdominal enhanced CT confirmed the curvatures of the anterior wall of the angular incisure as a thickening of the wall with a contrast effect. Lesions in the lower body of the stomach could not be identified. The abdominal mass was recognized as a malformed tumor with a maximum diameter of $13 \mathrm{~cm}$, and with a solid component that was lightly contrast-enhanced in its interior, which was located outside the stomach wall on the greater

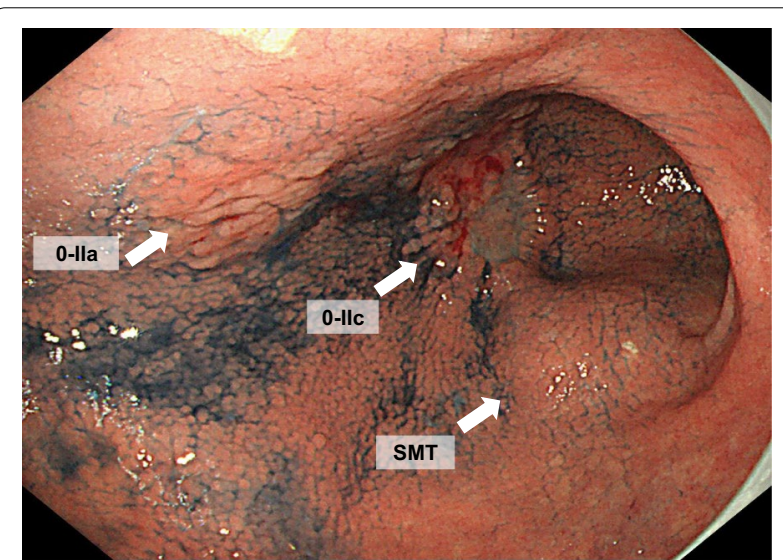

Fig. 1 Upper endoscopy: the 0-Ilc lesion, the 0-Ila lesion and the SMT like lesions (arrows) curvature side of the angular incisure. The boundary with the abdominal wall was unclear, and infiltration of the abdominal wall was suspected (Fig. 2). The preoperative diagnosis was GIST and two EGCs (M, Ant, 0-IIc, cT1bN0M0 cStage I; M, Ant, 0-IIa, cT1aNOM0 cStage I). Distal gastrectomy, D1 + lymph node dissection, and cholecystectomy were performed for the diagnosis of gastric GIST and EGCs. Reconstruction was performed by Billroth I. The giant tumor was identified as a large fist-shaped mass located in the greater curvature of the angular incisure that was adhered to the transverse colon and the transverse mesentery (Fig. 3a). The surgery time was $3 \mathrm{~h}$ and $45 \mathrm{~min}$, and the blood loss volume was $232 \mathrm{ml}$. Although the preoperative and intraoperative diagnosis was GIST, macroscopic findings of the resected specimen showed there was no continuity between the giant tumor and the stomach wall (Fig. 3b).

Histopathological findings are shown in Figs. 4, 5 and 6. The gastric lesions were diagnosed as two primary EGCs (M, Ant, type 0 -IIc, $32 \times 30 \mathrm{~mm}$, tub1 > tub2, pT1b (SM2), INFb, Ly1a, V1a, CY0, N1 (1/40), M0, pStage IB; and $\mathrm{M}$, Ant, type 0 -IIa + IIc, $10 \times 10 \mathrm{~mm}$, tub1, pT1a (M), Ly0, V0, CY0, N0, M0, pStage IA), and their histopathology was conventional tubular adenocarcinoma (Fig. 4a, b). The smaller lesion (type 0-IIa) was an intramucosal well-differentiated adenocarcinoma, while the larger one (type 0-IIc) infiltrated into a submucosal layer with lymphovascular invasion. The extragastric giant tumor was a moderately differentiated tubular adenocarcinoma with hemorrhage and extensive necrosis (Fig. 4c). Immunohistochemically, caudal type homeobox-2 (CDX-2) and cytokeratin 20 (CK20), representing an intestinal phenotype, were positive in both the gastric lesions and the giant tumor (Fig. 5). In addition, enteroblastic differentiation and a yolk sac tumor-like components with Schiller-Duval bodies were observed in the giant tumor. Approximately half of the cancer cells in the giant tumor were immunoreactive for SALL4 and some were AFPpositive (Fig. 6). Both human chorionic gonadotropin $(\beta$-hCG) and synaptophysin were negative. Except for the giant lymph node metastasis, no metastasis was observed in the other 39 dissected lymph nodes. Because the lymph node metastasis was solitary and large, a wholebody search was performed by colonoscopy and PET$\mathrm{CT}$ during the early postoperative period to detect other primary sites; however, there was no primary site other than the stomach. Therefore, we concluded that the giant tumor containing carcinoma with enteroblastic differentiation was a lymph node metastasis from the EGC.

Postoperative delayed gastric excretion occurred (Clavien-Dindo classification II), and decompression by gastric tube was need for 5 days. The patient was discharged on postoperative day 16 . Postoperative adjuvant 

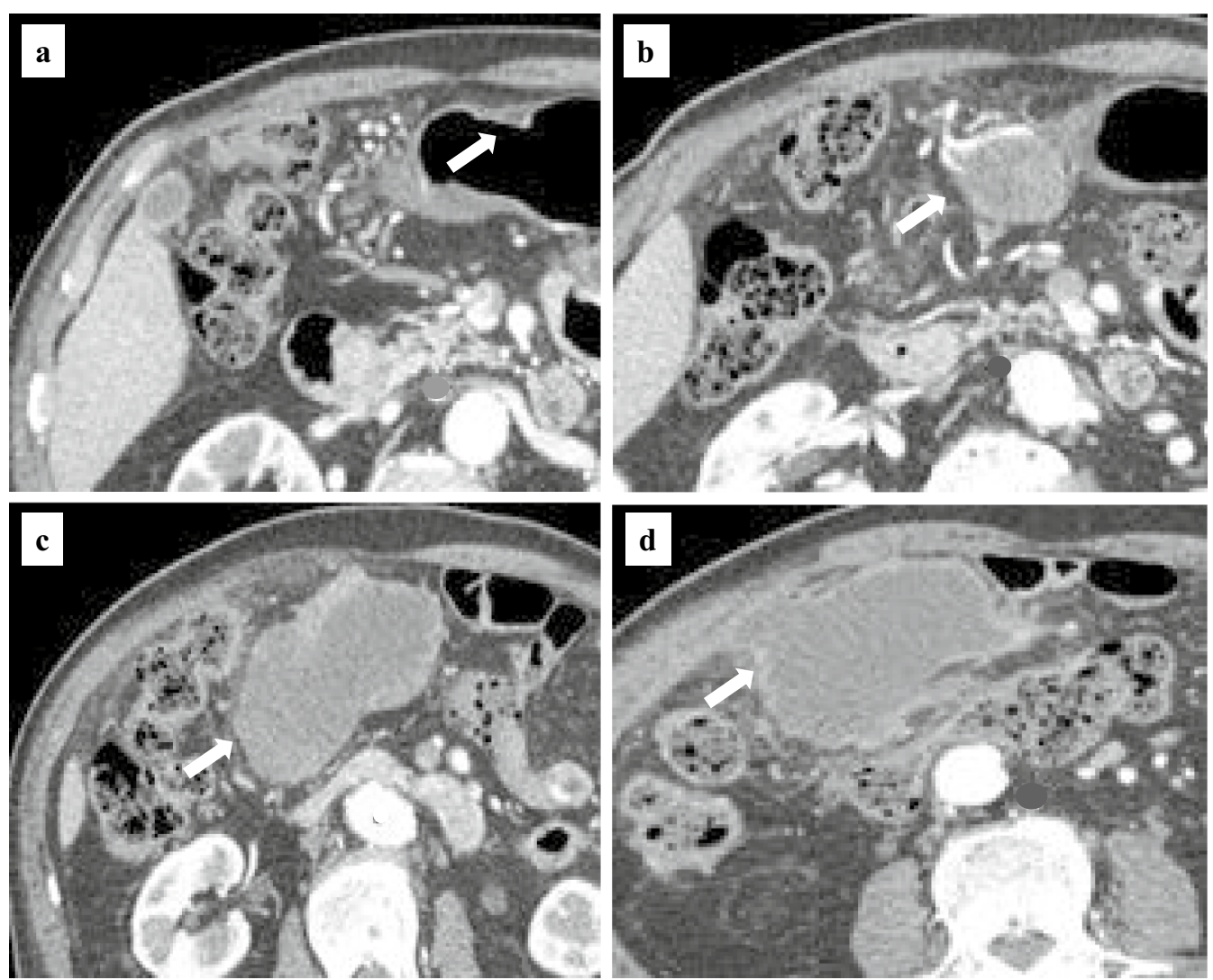

Fig. 2 Abdominal enhanced CT: the 0-Ilc lesion (a) and the giant tumor (b, c, d)
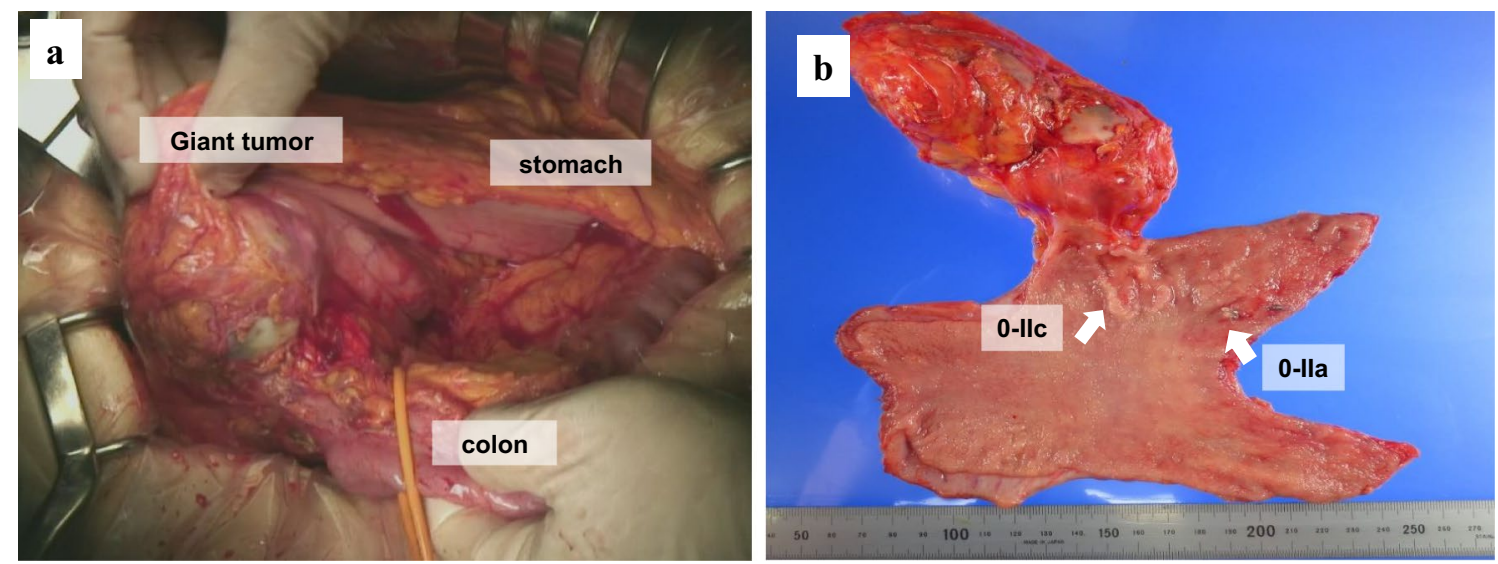

Fig. 3 Intraoperative findings (a) and macroscopic findings (b)

chemotherapy was not performed, and the patient was followed up. However, 6 months after surgery, his markers had risen. CT showed liver metastasis with a portal vein tumor plug, and SOX (S-1 + oxaliplatin) therapy was started (Fig. 7). The patient is alive 1 year after the surgery with stable disease (RECIST guideline version 1.1). 

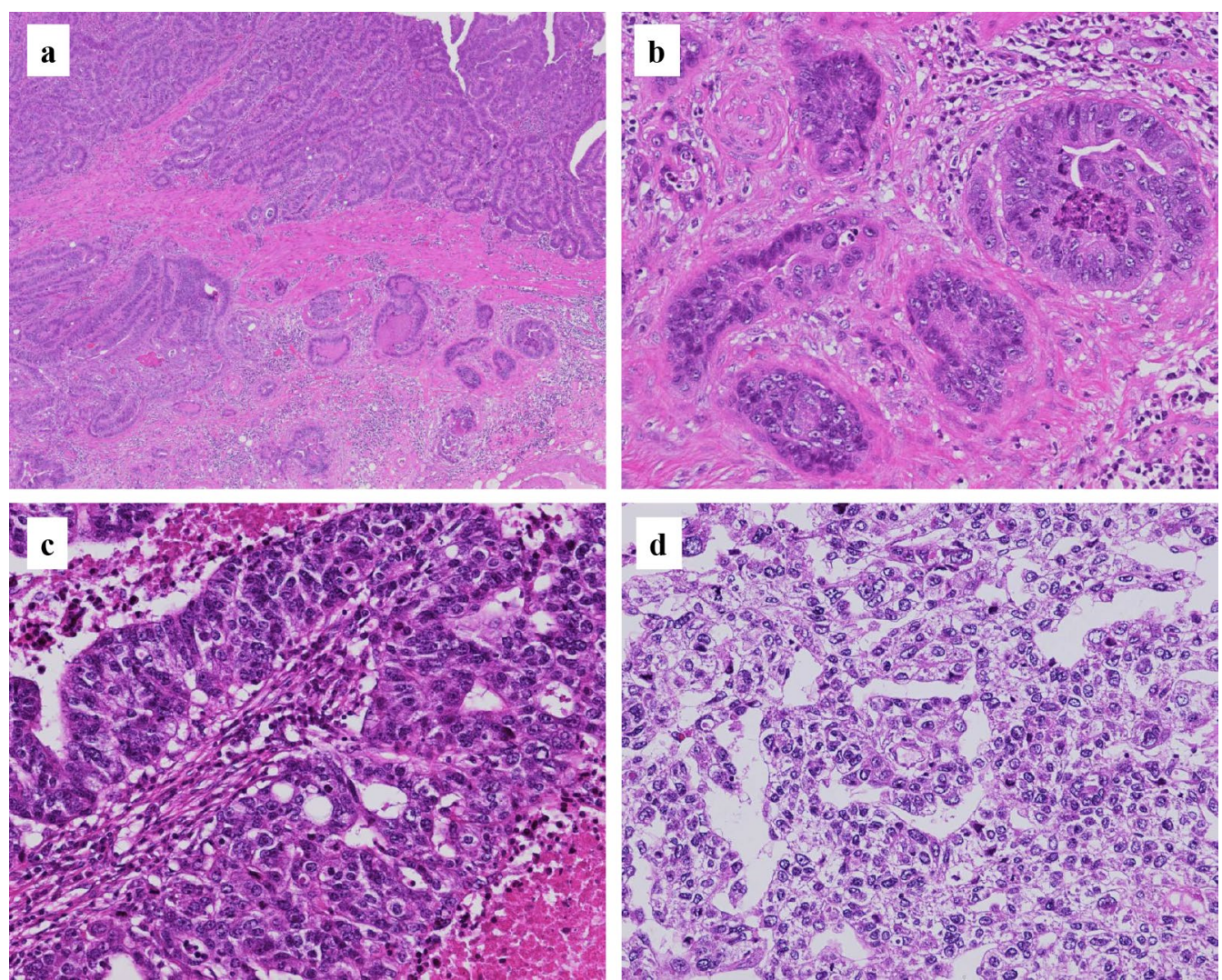

Fig. 4 Hematoxylin and eosin stain: the gastric type 0-Ilc lesion showed a tubular adenocarcinoma infiltrating into the submucosal layer (a, b). The metastatic adenocarcinoma with necrosis in the giant lymph node $\mathbf{( c )}$. The metastatic adenocarcinoma contained yolk sac tumor-like components with Schiller-Duval bodies (d) (original magnification, $\mathbf{a} \times 40, \mathbf{b}-\mathbf{d} \times 200$ )

\section{Discussion}

We experienced an extremely rare case of single giant lymph node metastasis from EGC. Pathological findings showed that the giant lymph node metastasis was moderately differentiated adenocarcinoma similar to that of primary EGC, and an intestinal phenotype was observed in both the primary and metastatic lesions by immunostaining. Because no primary lesion other than the stomach could be identified by postoperative colonoscopy and PET-CT examination, the giant tumor was diagnosed as lymph node metastasis of EGC.

We diagnosed the giant tumor as GIST only by CT and upper endoscopy. PET-CT was not considered due to poor glycemic control. MRI was not also considered for the definitive diagnosis of gastric submucosal tumor because of its lack of specificity in signal intensity and its pattern [5]. Because of concern regarding dissemination by needle biopsy, surgery was performed without biopsy.

In this case, we diagnosed EGC and GIST, and performed D1 + lymphnode dissection.

The decision was based on the idea that lymph node dissection was not necessary for GIST unless lymph node metastasis were apparent [6]. We determined that the giant tumor connected to the stomach wall and identified it as GIST from the preoperative and intraoperative findings. However macroscopic findings of the resected specimen showed there was no connection between the giant tumor and the stomach wall. It was suspected that the giant tumor could be an isolated tumor such as lymph node metastasis or primary omentum tumor. As a result, we could have decided the appropriate lymph node dissection range (D2 lymph node dissection) by frozen section during operation.

Four cases of EGC with a single giant lymph node metastasis of $5 \mathrm{~cm}$ or more were previously reported (Table 1), and in most cases the main complaint was abdominal symptoms resulting from the giant lymph node. No cases have been diagnosed as lymph node metastasis of gastric cancer before surgery, and diagnoses of pancreatic head, liver, and gastric submucosal tumors have been made. All cases were performed gastrectomy + lymph node dissection and alive without recurrence, although the follow-up period was shorter than 5 years $[2,7-9]$. 

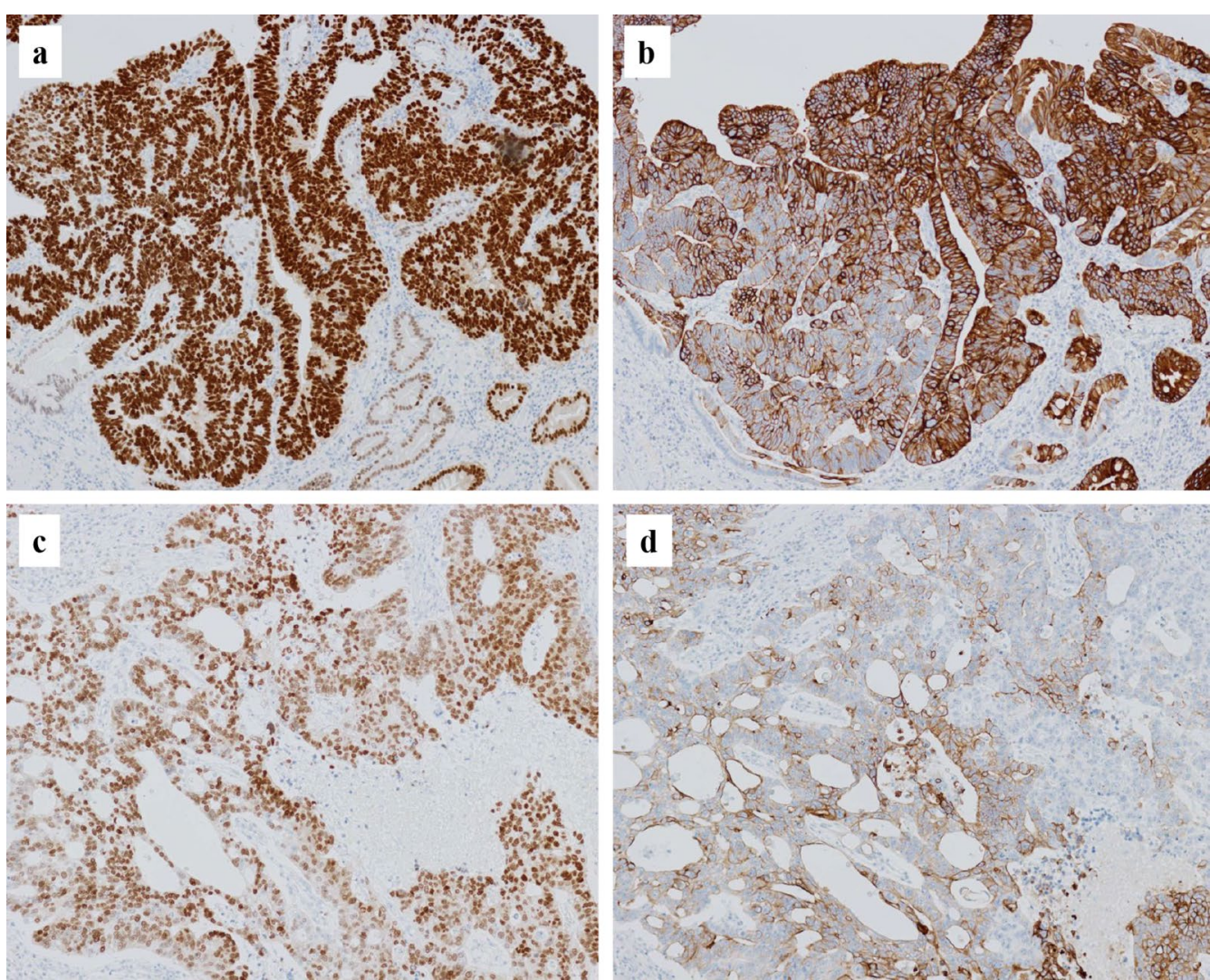

Fig. 5 Immunohistochemical staining of CDX-2 and CK20: CDX-2 expression of the 0-IIc lesion (a), and the giant tumor (c). CK20 expression of the 0 -Ilc lesion (b), and the giant tumor (d) (original magnification, each $\times 100$ )

The formation of large lymph nodes causes obstruction of lymph vessels and stagnation of lymph flow, and when cancer cells grow abnormally in one lymph node and most of the lymph nodes are occupied by lesions, it is believed that lymph flow is blocked and metastasis to the periphery is impeded. Therefore, it is thought that a good prognosis can be obtained by excision [10].

However, in this case, although it was a single lymph node metastasis, hematogenous metastasis to the liver occurred as early as 6 months after surgery. In the pathological diagnosis, the degree of atypia increased in the metastatic lymph nodes compared with the primary lesion, and the metastatic lymph nodes had a clear cytoplasm, a Schiller-Duval body-like structure that was not found in the primary lesion, and a yolk sac tumor-like carcinoma morphology. To date, retrodifferentiation to the yolk sac tumor or embryonic gastrointestinal tract during metastasis has been presumed to be the mechanism by which gastric cancer acquires fetal features and has not been found in the primary lesions with metastatic characteristics [3, 4]. In our case, retrodifferentiation to a yolk sac tumor during metastasis was considered a factor in the increased tumor malignancy.
In addition, in this case, the expression of SALL4, a fetal cancer protein, was observed in the primary lesion, although AFP expression was not observed. Recently, in addition to AFP, glypican-3 and SALL4 have been considered effective in diagnosing AFP-producing gastric cancer as fetal cancer proteins. Ushiku et al. reported that AFP production was observed in $8 \%$ of 338 cases of gastric cancer, and that all AFP-positive cases were also positive for glypican-3 and SALL4. Furthermore, in a study of 32 cases of AFP-producing gastric cancer, only $16 \%$ were positive for diffuse staining (50\% or more of tumor cells) in AFP, whereas it was as high as $56 \%$ positive for glypican-3 and 78\% for SALL4 [11]. In this case, although AFP expression and typical histology of yolk sac tumors were not observed in the primary lesion, SALL4 expression was positive, and the tumor had the characteristics of a fetal tumor. The most characteristic feature of gastric cancer with fetal components is that it is highly vascularly invasive and prone to liver metastasis. In a comparative study of fetal gastrointestinal epithelial-like cancer and conventional gastric cancer, lymphatic invasion ( $76 \%$ vs $41 \%$ ), venous invasion ( $72 \%$ vs $31 \%$ ), lymph node metastasis (69\% vs $38 \%)$, and liver metastasis $(31 \%$ 

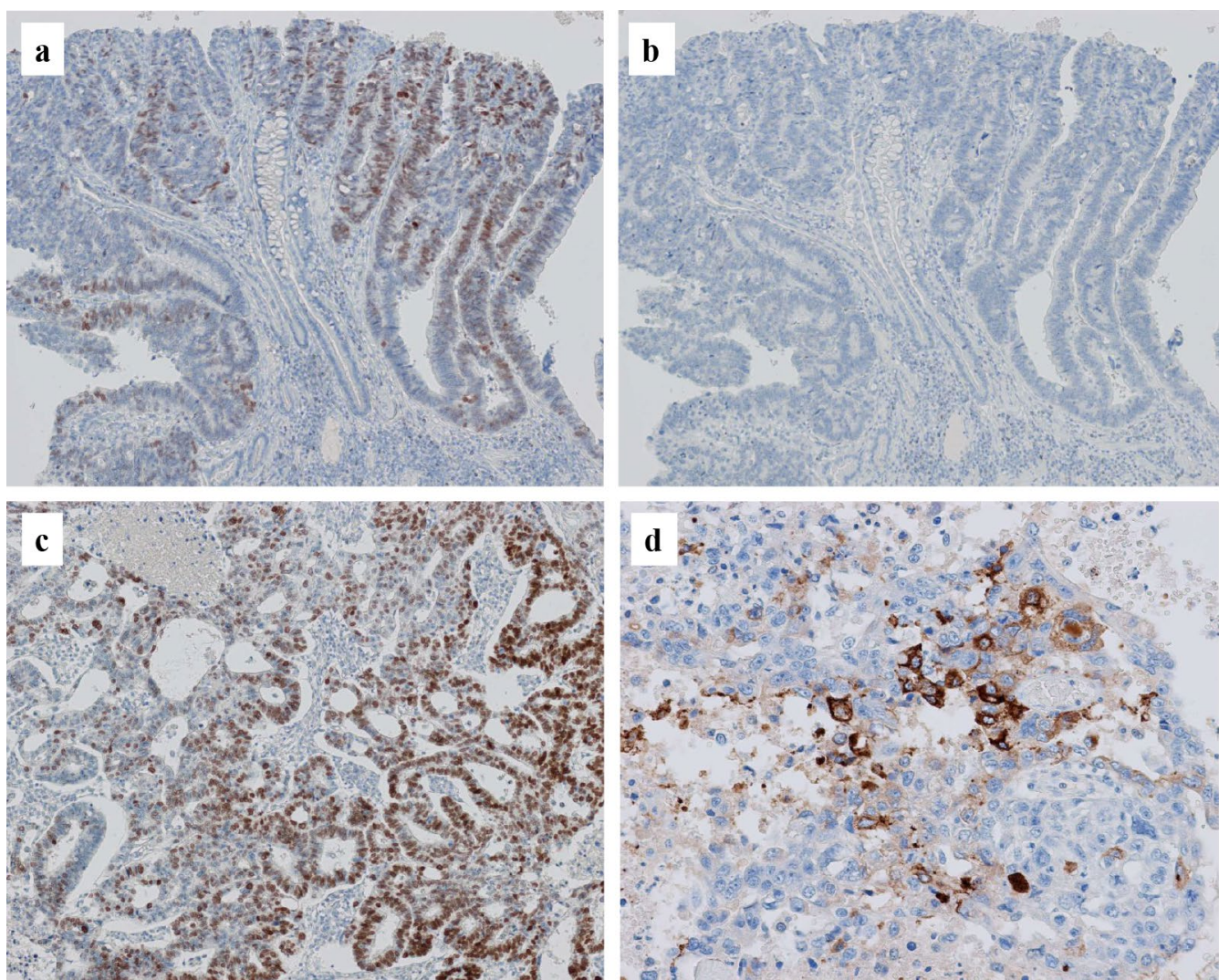

Fig. 6 Immunohistochemical staining of SALL4 and AFP: SALL4 expression of the 0-IIc lesion (a), and the giant tumor (c). AFP expression of the 0-IIc lesion (b), and the giant tumor (d) (original magnification, $\mathbf{a}: \times 40, \mathbf{b}: \times 40, \mathbf{c}: \times 100, \mathbf{d}: \times 200$ )
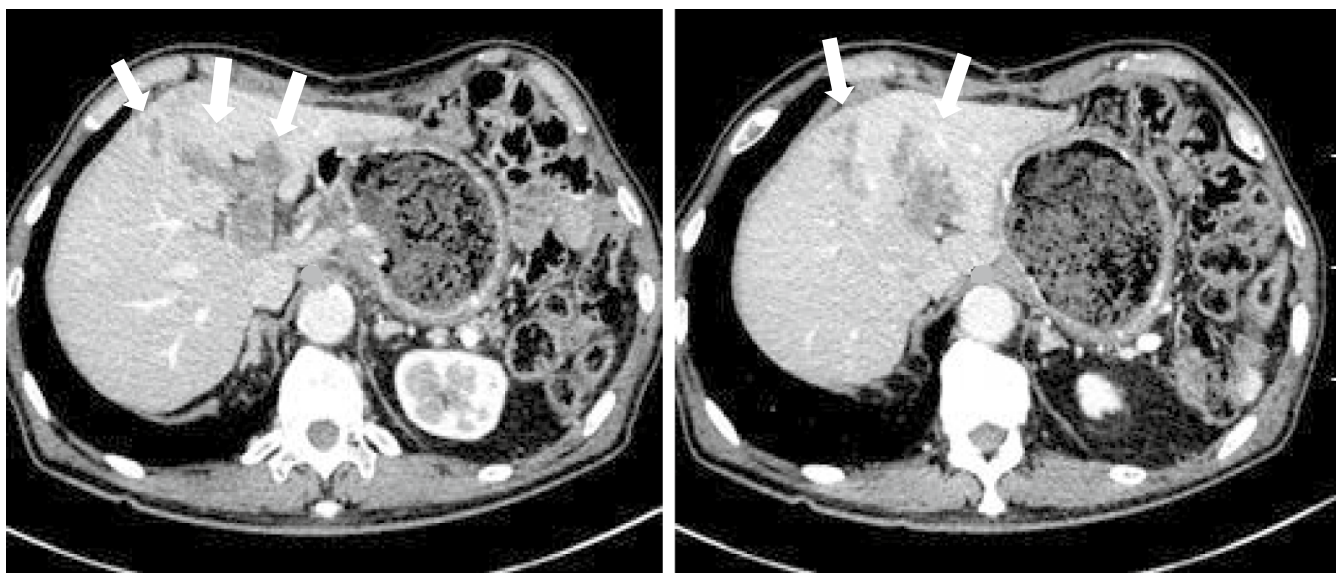

Fig. 7 Abdominal enhanced CT of 6 months after surgery: metastatic lesions are indicated by arrows

vs $6 \%$ ) were all significantly more frequent in carcinoma with enteroblastic differentiation. Regarding the prognosis, the 1 -year survival rate was $38.7 \%$, and was reported as $66.7 \%$ even in curative resection cases; thus, the prognosis is extremely poor compared with common typed gastric cancer [12]. In the current case, liver metastasis was observed early within 1 year after surgery and postoperative chemotherapy was introduced. Strict postoperative follow-up is essential for gastric cancer with fetal components, and, even in cases that could be resected, 
Table 1 Previous reports of EGC with a single giant lymph node metastasis

\begin{tabular}{|c|c|c|c|c|c|c|c|c|c|}
\hline Year & Writer & Age/sex & Chiefcomplaint & Preoperativediagnosis & $\begin{array}{l}\text { Postoperative } \\
\text { diagnosis } \\
\text { (differentiation, } \\
\text { depth, size: } \mathrm{cm} \text { ) }\end{array}$ & Procedure & LN & Adjuvant chemo & Prognosis \\
\hline 1987 & $\begin{array}{l}\text { Kamiya } \\
\text { et al. }\end{array}$ & $66 / M$ & $\begin{array}{l}\text { Upper abdomi- } \\
\text { nal pain }\end{array}$ & $\begin{array}{l}\text { Hepaticcyst adenocar- } \\
\text { cinoma }\end{array}$ & $\begin{array}{l}\text { L, Less } \\
\text { (tub1, m,0.8) } \\
\text { L,Ant } \\
\text { (tub1, m,0.4) }\end{array}$ & $\begin{array}{l}\text { Partial gas- } \\
\text { trectomy }\end{array}$ & $\begin{array}{l}\# 5 \\
5 \mathrm{~cm}\end{array}$ & - & $\begin{array}{l}2 \mathrm{Y} 1 \mathrm{M} \\
\text { Norec }\end{array}$ \\
\hline 1996 & $\begin{array}{l}\text { Terashima } \\
\text { et al. }\end{array}$ & $59 / F$ & Abdominalmass & $\begin{array}{l}\text { Gastriccancer and Pan- } \\
\text { creas or Liver tumor }\end{array}$ & $\begin{array}{l}\text { M, Less } \\
(\text { por, sm, -) }\end{array}$ & $\begin{array}{l}\text { Partial gas- } \\
\text { trectomy, } \\
\text { LateralHx, } \\
\text { DP, Sple- } \\
\text { nectomy }\end{array}$ & $\begin{array}{l}\# 3 \\
14 \mathrm{~cm}\end{array}$ & - & $\begin{array}{l}4 \text { YOM } \\
\text { No rec }\end{array}$ \\
\hline 2009 & Ishii et al. & $57 / M$ & - & $\begin{array}{l}\text { Gastriccancer and Pan- } \\
\text { creas tumor }\end{array}$ & $\begin{array}{l}\text { L,Post } \\
\text { (tub1,sm, 2.5) } \\
\text { L, Post } \\
\text { (tub1,sm, 0.7) } \\
\text { L,Ant } \\
\text { (tub1-por1, sm, } \\
0.6 \text { ) }\end{array}$ & $\mathrm{DG}, \mathrm{D} 1+$ & $\begin{array}{l}\# 6 \\
6 \mathrm{~cm}\end{array}$ & Unknown & $\begin{array}{l}1 \mathrm{Y} 10 \mathrm{M} \\
\text { No rec }\end{array}$ \\
\hline 2019 & $\begin{array}{l}\text { Umeda } \\
\text { et al. }\end{array}$ & $72 / \mathrm{M}$ & Abdominalmass & SMT & $\begin{array}{l}\text { M, Less } \\
\text { (tub1-2,mp, 2.5) }\end{array}$ & DG, D2 & $\begin{array}{l}\# 6 \\
8 \mathrm{~cm}\end{array}$ & $\begin{array}{l}+ \\
\text { Regimen:unknown }\end{array}$ & $\begin{array}{l}\text { 1Y8M } \\
\text { No rec }\end{array}$ \\
\hline 2021 & Our case & $78 / \mathrm{M}$ & Abdominalmass & SMT & $\begin{array}{l}\text { M, Ant } \\
\text { (tub1,sm, 3.0) } \\
\text { M, Ant } \\
\text { (tub1,sm, 1.5) }\end{array}$ & DG, D1+ & $\begin{array}{l}\# 4 \mathrm{~d} \\
13 \mathrm{~cm}\end{array}$ & - & $\begin{array}{l}6 \mathrm{M} \\
\text { Liver meta } \\
\rightarrow \text { SOX }\end{array}$ \\
\hline
\end{tabular}

postoperative adjuvant chemotherapy that is stronger than that for common typed gastric cancer may be considered.

\section{Conclusions}

We experienced a case of EGC with a single giant lymph node metastasis. Retrodifferentiation to the fetal gastrointestinal tract during metastasis was considered to be involved in the formation of giant lymph node metastasis and liver metastasis in this case.

\section{Abbreviations}

EGC: Early gastric cancer; CT: Computed tomography; GIST: Gastrointestinal stromal tumor; PET-CT: Positron emission tomography-computed tomography; AFP: a-Fetoprotein; SALL4: Spalt-like 4; SM: Submucosal layer; CEA: Carcinoembryonic antigen; LN: Lymph node; CDX-2: Caudal type homeobox-2; CK20: Cytokeratin 20; $\beta$-hCG: Human chorionic gonadotropin; SOX: S-1 + oxaliplatin; Hx: Hepatectomy; DP: Distal pancreatectomy; DG: Distal gastrectomy.

\section{Acknowledgements}

We thank H. Nikki March, PhD, from Edanz (https://jp.edanz.com/ac) for editing a draft of this manuscript.

\section{Authors' contributions}

SY, KS, MO and KK have made substantial contributions to the concept and design of the case report. MT and $\mathrm{HO}$ envisioned the study, participated in its design and coordination, and helped draft the manuscript. All authors read and approved the final manuscript.

\section{Funding}

Not applicable.

\section{Availability of data and materials}

The authors declare that all data in this article are available within the article.

\section{Declarations}

Ethics approval and consent to participate

Not applicable.

\section{Consent for publication}

Informed consent was obtained from the patient for publication of this report and the use of any accompanying images.

\section{Competing interests}

The authors declare that they have no competing interests.

\section{Author details}

${ }^{1}$ Institute of Ehime Prefectural Central Hospital, Department of Digestive Surgery, Matsuyama, Ehime 790-0024, Japan. ²Department of Pathology, Ehime Prefectural Central Hospital, Matsuyama 790-0024, Japan.

Received: 9 September 2021 Accepted: 9 November 2021

Published online: 18 November 2021

\section{References}

1. Suzuki $\mathrm{H}$, Endo $M$, Suzuki S, et al. A study of the lymphnode metastasis on early gastric cancer. J Jpn gastroenterol Surg. 1984;17:1517-26.

2. Umeda H, Yamano T, Kudo Y, et al. Gastric cancer with a giant lymph node metastasis - a case report-. J Jpan Surg Assoc. 2019:80:1492-6.

3. Tsutsumi Y. Differentiation of gastric cancer cells: analysis using immunohistochemistry and mucin histochemistry. Dig Dis Pathol. 1988;1:1-49.

4. Matsunou H, Konishi F, Roswelly EAJ, et al. Alpha-fetoprotein-producing gastric carcinoma with enteroblastic differentiation. Cancer. 1994;73:534-40. 
5. Nishida T, Hirota S, Yanagisawa A, et al. Clinical practice guidelines for gastrointestinal stromal tumor (GIST) in Japan: English version. Int J Clin Oncol. 2008;13(5):416-30.

6. Nishida T, Blay J-Y, Hirota S, et al. The standard diagnosis, treatment, and follow-up of gastrointestinal stromal tumors based on guidelines. Gastric Cancer. 2016;19(1):3-14.

7. Kamiya J, Nimura Y, Hayakawa N, et al. A case of double mucosal cancers of the stomach with a large and necrotic lymph node metastasis. J Jpn gastroenterol Surg. 1987;20:808-11.

8. Terashima S, Ono T, Ito F, et al. Early stomach cancer with significant lymph node involvement. Jpn Cancer Clinics. 1996;42:293-7.

9. Ishii M, Miyazaki T, Ishibashi K, et al. Infrapyloric lymph node metastasis 6 $\mathrm{cm}$ in diameter in a patient with multiple mucosal cancers of the stomach: a case report. Jpn College of Surgeons. 2009;34:61-6.
10. Miyagawa $\mathrm{H}$, Muto I. A case of gastric cancer presented with rupture of metastatic lymph node. J Jpn Surg Assoc. 1999;60:715-20.

11. Ushiku T, Hukayama M. Fetal Type Gastric Carcinoma and its Markers, SALL4, Glypican 3, and AFP. Stomach Intestine. 2010; 45:1950-8 (In Japanese with English abstract)

12. Murakami T, Yao T, Mitomi H, et al. Clinicopathologic and immunohistochemical characteristics of gastric adenocarcinoma with enteroblastic differentiation : a study of 29 cases. Gastric Cancer. 2016;19:498-507.

\section{Publisher's Note}

Springer Nature remains neutral with regard to jurisdictional claims in published maps and institutional affiliations.

\section{Submit your manuscript to a SpringerOpen ${ }^{\circ}$ journal and benefit from:}

- Convenient online submission

- Rigorous peer review

- Open access: articles freely available online

- High visibility within the field

- Retaining the copyright to your article

Submit your next manuscript at $\boldsymbol{\nabla}$ springeropen.com 